\title{
Membrane steroid-binding protein 1 (MSBP1) negatively regulates brassinosteroid signaling by enhancing the endocytosis of BAK1
}

\author{
Li Song ${ }^{1}$, Qiu-Ming Shi ${ }^{1}$, Xiao-Hua Yang ${ }^{1,2}$, Zhi-Hong Xu ${ }^{1}$, Hong-Wei Xue ${ }^{1}$

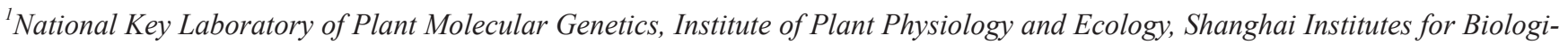 \\ cal Sciences, Chinese Academy of Sciences, 300 Fenglin Road, Shanghai 200032, China
}

Brassinosteroids (BRs) are perceived by transmembrane receptors and play vital roles in plant growth and development, as well as cell in responses to environmental stimuli. The transmembrane receptor BRI1 can directly bind to brassinolide (BL), and BAK1 interacts with BRI1 to enhance the BRI1-mediated BR signaling. Our previous studies indicated that a membrane steroid-binding protein 1 (MSBP1) could bind to BL in vitro and is negatively involved in BR signaling. To further elucidate the underlying mechanism, we here show that MSBP1 specifically interacts with the extracellular domain of BAK1 in vivo in a BL-independent manner. Suppressed cell expansion and BR responses by increased expression of MSBP1 can be recovered by overexpressing BAK1 or its intracellular kinase domain, suggesting that MSBP1 may suppress BR signaling through interacting with BAK1. Subcellular localization studies revealed that both MSBP1 and BAK1 are localized to plasma membrane and endocytic vesicles and MSBP1 accelerates BAK1 endocytosis, which results in suppressed BR signaling by shifting the equilibrium of BAK1 toward endosomes. Indeed, enhanced MSBP1 expression reduces the interaction between BRI1 and BAK1 in vivo, demonstrating that MSBP1 acts as a negative factor at an early step of the BR signaling pathway.

Keywords: MSBP1, BR signaling, BAK1, endocytosiss

Cell Research (2009) 19:864-876. doi: 10.1038/cr.2009.66; published online 16 June 2009

\section{Introduction}

Brassinosteroids (BRs) play crucial roles in multiple processes of plant growth and development, including stem elongation, leaf expansion, vascular differentiation, senescence and stress tolerance [1]. Defects in BR biosynthesis and signaling result in dwarfism, dark-green and rounded leaves, delayed development, reduced fertility and abnormal vascular pattern of plants [2-6]. Up to now, several key components of the BR signaling pathway have been identified through molecular and genetics studies.

\footnotetext{
Correspondence: Hong-Wei Xue

Tel: +86-21-54924059; Fax: +86-21-54924060

E-mail: hwxue@sibs.ac.cn

${ }^{2}$ Current address: University of Maryland Biotechnology Institute, 9600 Gudelsky Drive, Rockville, MD 20850, USA

Received 1 December 2008; revised 22 December 2008; accepted 26 December 2008; published online 16 June 2009
}

In Arabidopsis, BR signal is perceived by the extracellular domain of the transmembrane receptor BRI1 [7-10]. BAK1, a BRI1 co-receptor, has a similar expression pattern and subcellular localization with BRI1, and serves as a signal transducer after the BR signal is recognized by BRI1. Although overexpression of BAK1 could rescue the phenotypes caused by BRII deficiency, BAK1 had no effects on the binding activity of BRI1 to BR molecules $[11,12]$.

Homodimerization of BRI1 leads to the self-inhibition of BRI1 activity and heterodimerization with BAK1 will activate BRI1, although the correlation between BR molecule and heterodimerization of BRI1-BAK1 is unclear. It has been shown that binding of ligand induces the heterodimerization of BRI1 and BAK1, which then initiates the BR signal transduction in Arabidopsis [13, 14]. However, BR-independent BRI1-BAK1 dimerization also occurs in cowpea protoplast and BAK1 stimulates the endocytosis of BRI1, suggesting that receptor endocytosis might be required for BR signaling [15]. 
Many other components of the BR signaling pathway transducing the BR signal from the cell surface to the nucleus have been genetically identified. The membranelocalized proteins BSKs interact with BRI1 in vivo and activate the downstream signaling of BRI1 [16]. On the opposite, another BRI1-interacting protein, BKI1, negatively regulates BR signaling by limiting the association of BRI1 and BAK1 [17]. BIN2 is a GSK3-like protein and negatively controls BR signaling by phosphorylating two nuclear components BES1 and BZR1 [18, 19]. As nuclear transcription factors, the unphosphorylated BES1 and BZR1 can activate the expressions of BRresponsive genes to promote plant growth (BZR1 also inhibits the expression of BR biosynthetic genes), while the phosphorylated BES1 loses its DNA-binding activity to the BR-responsive genes, and phosphorylated BZR1 is rapidly degraded by proteasome to relieve its feedback inhibition of several BR biosynthetic genes [20-24].

Recent studies showed that endosomes play important roles in the signal transduction process. In animals, cholesterol functions extensively in various developmental processes [25], and cholesterol-dependent vesicle transport contributes to the establishment of cell polarity in Caenorhabditis [26] and pattern formation during Drosophila wing development [27]. In plants, PIN proteins modulate auxin efflux by their polar localization, and recycle between the plasma membrane and endosomal compartments in a vesicle trafficking-dependent manner [28-30]. FLS2, a leucine-rich-repeat (LRR) receptor-like kinase involved in perceiving bacterial elicitor flagellin in Arabidopsis, exhibits ligand-dependent endocytosis $[31,32]$. In addition, BAK1 and FLS2 form a complex in a specific ligand-dependent manner to initiate the innate immunity signaling, which is independent of BR signaling $[33,34]$.

Recent studies showed that vesicle cycling also plays crucial roles in BR signal transduction. In Arabidopsis root meristems, BRI1 is localized at plasma membrane and endosomal compartments in a ligand-independent manner, and increased endosomal localization of BRI1 could enhance BR signaling and genomic responses [35]. In cowpea protoplasts, both BRI1 and BAK1 are localized at plasma membrane and endocytic compartments, and their interactions occur in the endocytic compartments, not just restricted to the sub-domains of plasma membrane. BAK 1 accelerates BRI1 endocytosis to stimulate BR signaling by shifting the equilibrium of the membrane BRI1 toward endosomes [15]. SERK1, the homolog of BAK1 (SERK3), is localized in endosomal compartments [36] and is involved in BR signaling by interacting with BRI1 and BAK1 [37], indicating that endocytosis may play a critical role in receptor recycling and function.

In animal cells, the plasma steroid-binding proteins (SBPs) function as receptors of steroid hormones to initiate the cellular responses [38-41]. The plant membranelocalized SBP, membrane steroid-binding protein 1 (MSBP1), binds to 24-epi-brassinolide (BL) in vitro with a lower affinity, compared to BRI1, and negatively controls cell elongation. Arabidopsis plants with enhanced expression of MSBPI have increased steroid moleculebinding capacity and suppressed response to exogenous $\mathrm{BL}$, indicating a negative role of MSBP1 in BR signaling [42].

Until now, how SBPs function in BR signaling regulation is still unclear. We here show that MSBP1 enhances BAK1 endocytosis by interacting with its extracellular region, resulting in the reduced association of BRI1 and BAK1, and suppressed BR signaling.

\section{Results}

MSBP1 specifically interacts with the extracellular domain of BAK1 in a BL-independent manner

To investigate the possible interactions between MSBP1 and other factors, we first tested the associations of MSBP1 with the transmembrane receptors BRI1 and BAK1. A yeast two-hybrid analysis showed that MSBP1 did not interact with BRI1, nor with the intracellular kinase domain of BAK1, but did associate specifically with the BAK1 extracellular domain (Figure 1A). This is consistent with the computational prediction showing that the steroid-binding region of MSBP1 is probably situated outside of the plasma membrane (Supplementary information, Figure S1, by TMHMM program $[43,44]$ ), and suggests that MSBP1 might act as a co-receptor with BAK1. Analysis employing XA21, another member of the LRR-RLK subfamily (involved in the resistance to the bacterial blight pathogen in rice, [45]), further revealed that there is no association between MSBP1 and the extracellular domain of XA21 (Figure 1A), indicating a specific interaction between MSBP1 and the BAK1 extracellular region.

Co-immunoprecipitation (Co-IP) studies further confirmed the interaction between MSBP1 and BAK1 extracellular domain in yeast cells (Figure 1B). In addition, using the antibody specific to MSBP1, we were able to detect the interaction between endogenous MSBP1 and BAK1 in plant cells, while there was no detectable interaction between the intracellular kinase domain of BAK1 and MSBP1 (Figure 1C), confirming the specific interaction between the BAK1 extracellular region and MSBP1 in vivo.

Previous studies showed that the association and ki- 
nase activities of BRI1 and BAK1 in yeast cells were not affected by exogenous BL [46]. In a similar manner, $\beta$-galactosidase assays showed that supplement of BL did not influence the intensity of MSBP1-BAK1 interaction (Figure 1D), which was further demonstrated by observing the MSBP1-BAK1 interaction in plant cells under exogenously applied BL (Figure 1E).

Enhanced BAK1 expression rescues the suppressed BR signaling caused by MSBP1 overexpression

Our previous studies showed that transgenic plants overexpressing MSBP1 had suppressed BR responses,
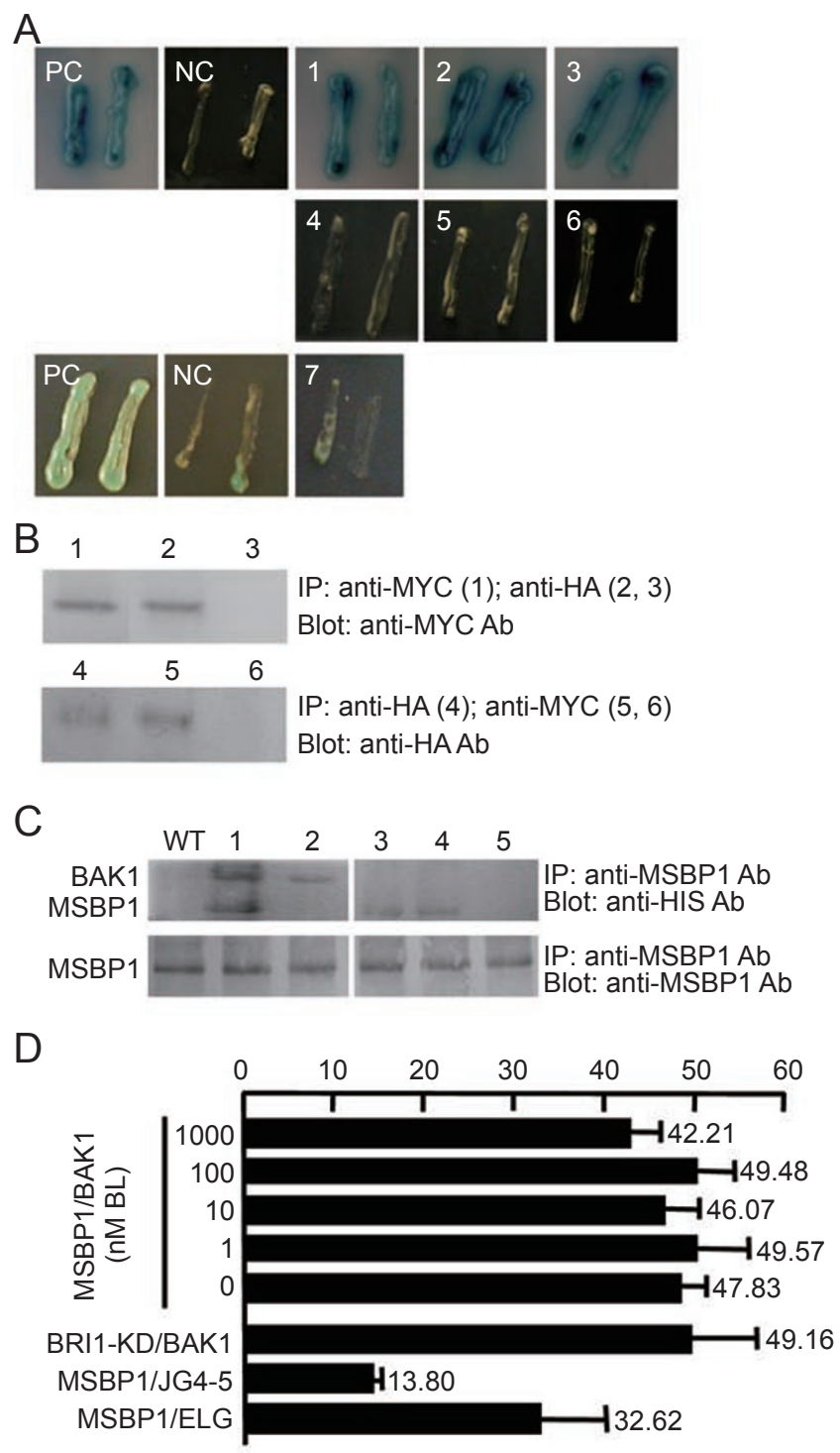

$E$

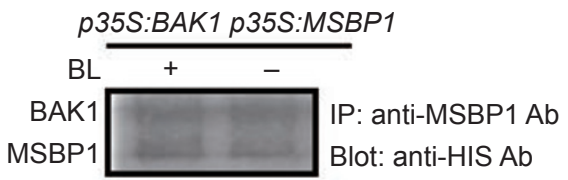

suggesting that MSBP1 had a negative effect on BR signaling. Plants with increased expression of MSBP1 were thus employed to study the mechanism by which MSBP1 suppresses BR signaling. Based on the interaction between MSBP1 and the extracellular domain of BAK1, we generated transgenic plants overexpressing BAK1 or its intracellular kinase domain in wild-type (WT) or p35S:MSBP1 backgrounds (Supplementary information, Figure S2). Phenotypic analysis showed that the suppressed cell elongation/expansion and responses to exogenous BL of $p 35 S: M S B P 1$ seedlings could be rescued by overexpressing BAK1, especially its intracellular kinase domain. Measurement of cell numbers revealed that overexpression of BAK1 or its kinase domain significantly stimulated the cell expansion in $p 35 S: M S B P 1$ and WT plants (Figure 2A). This effect was more evi-

Figure 1 MSBP1 interacts with the extracellular domain of BAK1 in a BL-independent manner. (A) Yeast two-hybrid analysis revealed the interaction between MSBP1 and extracellular domain (EC) of BAK1, but not its intracellular kinase domain (KD), nor BRI1 or the extracellular domain of rice XA21 protein. Growth of yeast cells containing various combinations of constructs was tested on SD-Leu/-Trp/-His/-Ade dropout medium. The combined constructs include: PC (positive control, pGBKT7-53 and pGADT7-T); NC (negative control, pGBKT7-Lam and pGADT7-T); 1, BRI1-KD/BAK1-KD; 2, MSBP1/BAK1-EC; 3, MSBP1/BAK1; 4, BRI1-EC/BAK1-EC; 5, BRI1-KD/BAK1-EC; 6, MSBP1/BAK1-KD and 7, MSBP1/XA21-EC. The relevant coding region is in-frame fused to that of the GAL4-binding domain (BD) or activation domain (AD). (B) Co-immunoprecipitation (Co-IP) analysis confirmed the interaction between the extracellular domain of BAK1 and MSBP1 in yeast cells. MSBP1 and BAK1 were tagged with $M Y C$ and $\mathrm{HA}$, and detected with antiMYC (1) or anti-HA (4) antibodies, respectively. BAK1 could be co-immunoprecipitated with $\operatorname{MSBP} 1(2,5) .3$ and 6 , the empty negative controls. (C) Co-IP analysis confirmed the interaction between the extracellular domain of BAK1 and MSBP1 in plant cells. Total membrane proteins were extracted from WT or homozygous transgenic plants of $1, p 35 S: B A K 1$ p35S:MSBP1; 2 , p35S:BAK1; 3, p35S:MSBP1; 4, p35S:BAK1-KD p35S:MSBP1 and 5, p35S:BAK1-KD. Both MSBP1 and BAK1 were tagged with HIS. Bottom line showed that equal amount of proteins was loaded. (D) Quantitative analysis employing yeast LexA twohybrid system reveals the interaction intensity of MSBP1 and BAK1 was not regulated upon BL treatment. In addition, the association between MSBP1 and ELG (a mutant form of BAK1) was decreased. Intracellular kinase domain of BRI1 and whole BAK1, MSBP1 and JG4-5 are used as positive and negative controls, respectively. Error bars represent standard deviations (SD). (E) Western blot analysis confirmed the unaltered interaction of MSBP1 and BAK1 in the presence of exogenous BL. Seedlings were treated with $100 \mathrm{nM} \mathrm{BL}$ for $8 \mathrm{~h}$ and anti-HIS antibody was used for protein detection, after Co-IP with antiMSBP1 antibody. 
dent when observing the 5th rosette leaves of 5-weekold plants, with the intracellular kinase domain of BAK1 showing a more obvious effect and the full-length BAK1 showing a less effect (Supplementary information, Figure S3).

To elucidate the role of MSBP1-BAK1 interaction in BR signaling, hypocotyl lengths of seedlings overexpressing MSBP1 or BAK1 under BR treatment were measured. Results showed that bril or bakl mutants and $p 35 S: M S B P 1$ plants exhibited decreased response
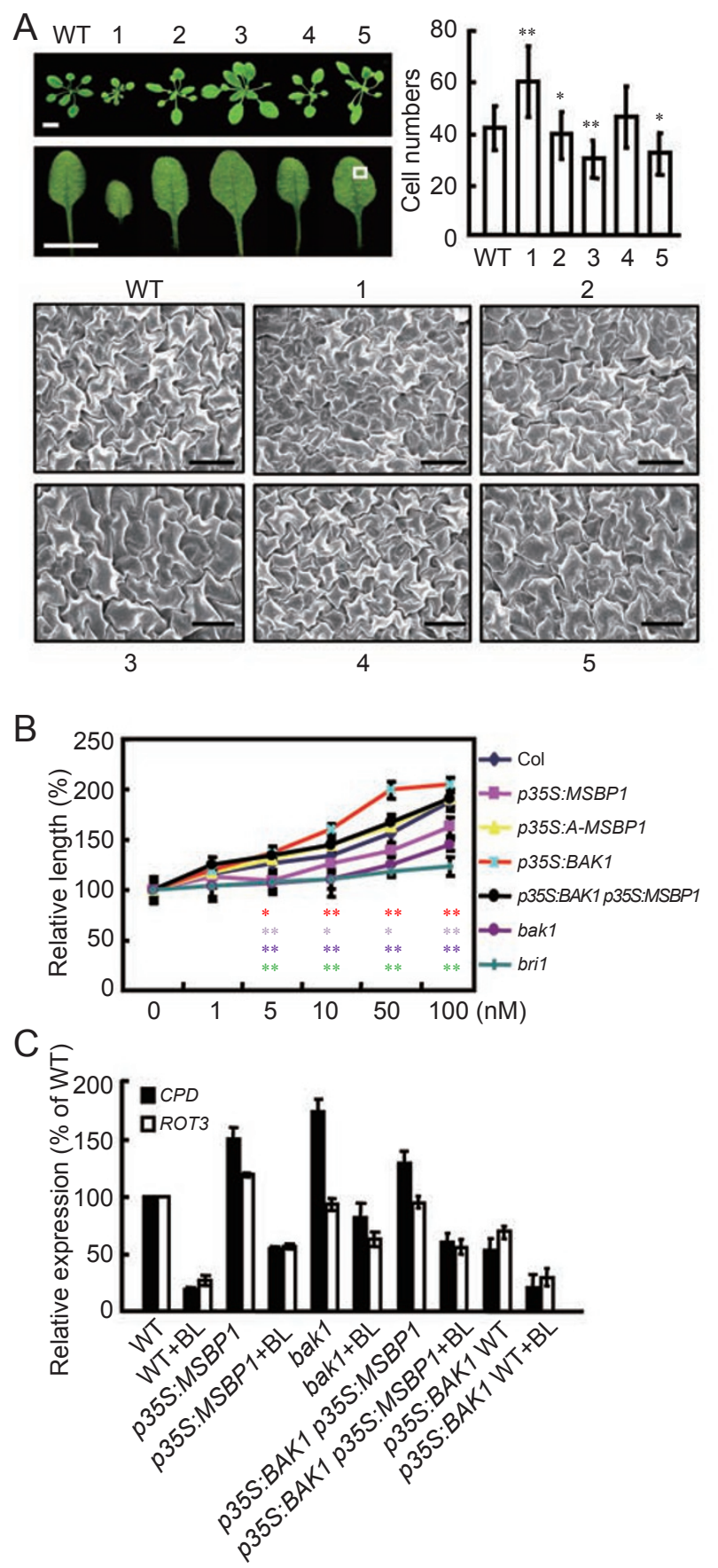

to exogenous $\mathrm{BL}$, and overexpression of BAK1 in WT background resulted in enhanced sensitivity to BL. Compared with WT, the reduced response of $135 S: M S B P 1$ seedlings to exogenous BL (5-100 nM) could be rescued by enhanced expression of BAK1 under light conditions (Figure 2B). These results support the idea that MSBP1BAK1 interaction plays an important role in regulating BR responses.

It was shown that the homeostasis of BR concentration was maintained through feedback regulation of the expression of multiple BR-biosynthetic genes [47-49]. Signaling-defective mutants, bril and bakl, showed reduced inhibition of transcription of BR-biosynthesis genes by BL $[6,11]$. We, therefore, tested whether overexpression of MSBP1 altered the expression of BR-biosynthetic genes using quantitative real-time reverse transcription PCR. Compared with that in WT, the expression level of $C P D$ was dramatically increased (by $49 \%$ ) and ROT3 slightly increased (by 17\%) in p35S:MSBP1 plants. After treatment with exogenous BL (100 nM, $3 \mathrm{~h})$, relative expression levels of $C P D$ and $R O T 3$ in p35S:MSBP 1 plants were reduced to $53 \%$ or $56 \%$, and the reduction was less than that in WT (CPD to $19 \%$; ROT3 to $26 \%$, Figure 2C), consistent with the suppressed BR signaling in $p 35 S: M S B P 1$ plants. Further analysis showed that the expression of $C P D$ and ROT3 was more severely suppressed by BL in WT plants overexpressing

Figure 2 The negative effect of MSBP1 on BR signaling is rescued by overexpression of BAK1 or its intracellular kinase domain. (A) Compared to WT, reduced cell expansion by overexpressing MSBP1 (1) was rescued by enhanced expression of BAK1 (4) or its intercellular KD (p35S:BAK1-KD p35S:MSBP1, 5). Overexpression of BAK1 (2) or its intercellular KD (p35S:BAK1-KD, 3) in WT plants result in even enlarged leaves (left panel, bar $=1 \mathrm{~cm}$ ). Rosette leaves of 3-week-old plants were sampled and the adaxial epidermis near the midvein (squared region) was examined under scanning electron microscopy (bar $=50 \mu \mathrm{m})$. Cell numbers of unit area $(\sim 0.063$ $\mathrm{mm}^{2}$ ) were measured and statistically analyzed using a onetailed Student's $t$-test $(*, P<0.05$; **, $P<0.01$ ) (right panel). Error bars stand for SE. (B) Overexpression of BAK1 leads to rescued response of $p 35 S: M S B P 1$ plants to BL in light. Seedlings were treated with different concentrations of $B L$. Lengths of hypocotyls (7-day-old seedlings) were measured and relative elongation was calculated and presented as average \pm SD $(n>$ 40). Statistical analysis was carried out through a two-tailed Student's $t$-test $(*, P<0.05$; **, $P<0.01$; different colors showed relevant plants compared with WT). (C) Quantitative RT-PCR analyses indicate that BR-biosynthesis-related genes $C P D$ and ROT3 were increased in bak1 mutant and p35S:MSBP1 plants, and were less suppressed under exogenously BL. qRT-PCR was performed using 1-week-old Arabidopsis seedling in the absence or presence of BL (100 nM, 3 h). Error bars stand for SD. 
$B A K 1$ than in $p 35 S: M S B P 1$ plants overexpressing $B A K 1$ (Figure 2C), confirming the negative role of MSBP1 in BR signaling.

Overexpression of MSBP1 partially suppresses BR hypersensitivity of elg mutant under darkness

Previously identified elg mutant, which harbors a mutation in the extracellular region of BAK1, confers BR hypersensitivity [47]. The interaction between MSBP1 and the mutant BAK1 protein was weaker than WT protein (Figure 1D), and the expression of BR biosynthesis genes was more severely suppressed by $\mathrm{BL}$ in the elg mutant (Figure 3A). These observations suggest that enhanced responses of elg to BL were possibly due to the reduced MSBP1-BAK1 interaction, which leads to the reduced inhibition of BAK1 activity by MSBP1.

To test this hypothesis, MSBP1 was overexpressed in elg and bakl mutants (Supplementary information, Figure S4). Phenotypic observation showed that MSBP1 overexpression in elg background caused a reduction of the hypersensitive response of hypocotyls to BR in dark, but did not affect the root growth under light conditions. In addition, overexpressing $M S B P 1$ in bakl mutant did not alter its responses to BR at all (Figure 3B and Supplementary information, Figure S5), strongly supporting the idea that MSBP1 regulates BR signaling through interacting with BAK1.

MSBP1-BAK1 association stimulates the endocytosis of $B A K 1$

It was previously shown that BRI1 and BAK1 were localized at plasma membrane and in endocytic compartments of protoplasts, and BAK1 accelerated the endocytosis of BRI1 [15]. Interestingly, analyses of protein localization through transient expression in Arabidopsis mesophyll protoplasts showed that MSBP1 was localized not only at the plasma membrane (Figure 4A, 3), but also in the vesicle-like compartments, confirmed by staining with FM4-64 (Figures 4A, 5-7). This result suggested that MSBP1 was also subjected to endocytosis. In addition, comparison with the localization of Golgi-localized $\mathrm{N}$-a-sialyltransferase (N-ST-YFP, fused to yellow fluorescent protein, [48]) showed that the MSBP1 vesicles do not represent proteins in transit from Golgi membranes (Supplementary information, Figure S6), supporting that the origin of MSBP1 vesicles is plasma membrane. BAK1 was localized to the plasma membrane and vesicles as well (Figure 4A, 4), in agreement with its location in cowpea protoplasts [15].

Further co-expression analysis revealed the colocalization of BAK1 and MSBP1 (Figures 4A, 8-11) in vesicles and at plasma membrane. Moreover, enhanced

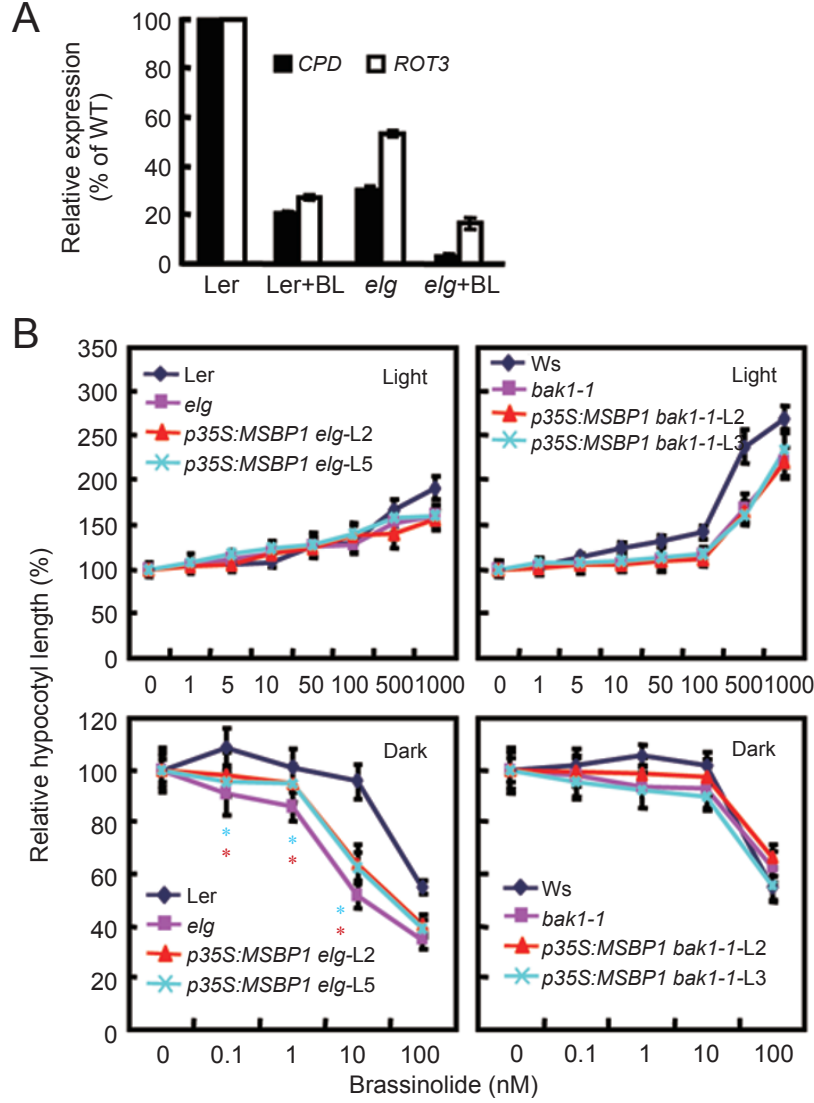

Figure 3 MSBP1 overexpression partially rescues the phenotype of elg, but not bak1. (A) Expression of CPD and ROT3 genes were suppressed in elg mutant in the absence or presence of BL. Error bars stand for SE. (B) Relative hypocotyl elongation of elg or bak1 seedlings, or those with overexpressed MSBP1 under different concentrations of exogenous $B L$. Lengths of hypocotyls (7-day-old seedlings in light or 4-day-old seedlings in dark) were measured and presented as average $\pm S D(n>40)$, and statistically calculated through a twotailed Student's $t$-test $(*, P<0.05$; different color showed relevant plants compared with WT).

endocytosis and distinct accumulation of BAK1 were observed (Figure 4B), indicating a role of MSBP1 in stimulating BAK1 endocytosis. Quantitative determination of BAK1-CFP fluorescence in mesophyll cells indicated that $\sim 72 \%$ of total BAK1 were detected in cytoplast under MSBP1 overexpression, in comparison with $\sim 55 \%$ of total BAK1 in cytoplast in control cells, confirming the altered localization of BAK1 upon overexpression of MSBP1. This is further supported by studying the dynamics of BAK1-MSBP1 interaction in onion epidermal cells. When MSBP1 and BAK1 were coexpressed, 35$41 \%$ of total BAK 1 were found in cytoplast, in comparison with $\sim 23 \%$ of total BAK1 in cytoplast in control cells (Supplementary information, Figure S7). 

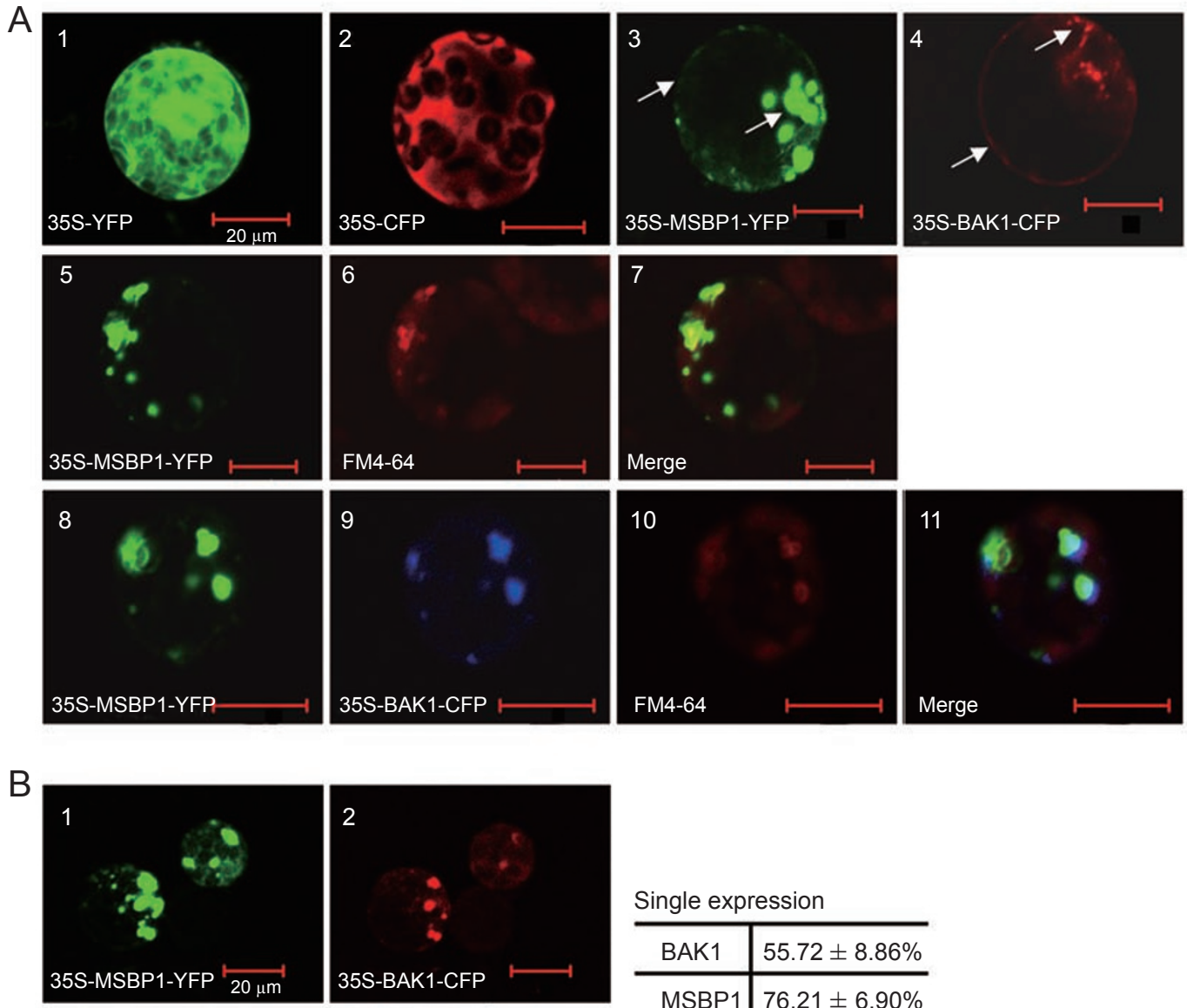

Single expression
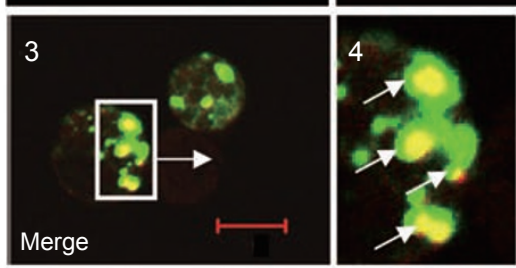

\begin{tabular}{c|c}
\hline BAK1 & $55.72 \pm 8.86 \%$ \\
\hline MSBP1 & $76.21 \pm 6.90 \%$ \\
\hline
\end{tabular}

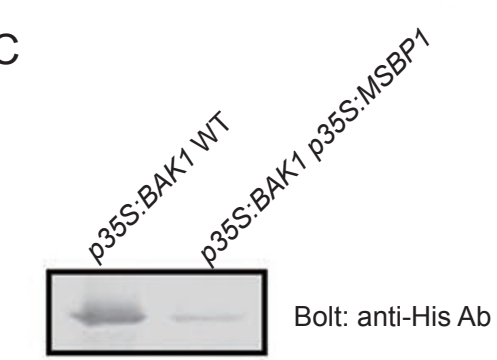

Figure 4 BAK1-MSBP1 association enhances the endocytosis of BAK1 in Arabidopsis mesophyll protoplasts. (A) Compared to the diffused expressions of EYFP (1) and ECFP (2) in cells, observation on the fluorescences of 35S-MSBP1-EYFP revealed that MSBP1 was localized at both plasma membrane and endosomes (3, arrows), which is confirmed by observing the internalization of FM4-64 (5-7). Similarly, BAK1 was localized at both plasma membrane and endosomes as well (4, arrows). Co-localization of MSBP1 and BAK1 in the endosomes was confirmed by FM4-64 staining $(8-11)$. Bar = 20 $\mu \mathrm{m}$. (B) Co-expression of MSBP1 and BAK1 resulted in the accelerated BAK1 endocytosis (1-3). The squared region was enlarged to highlight the BAK1 localizations (4). Bar $=20 \mu \mathrm{m}$. Statistical analysis on the fluorescence intensity in cytoplast indicated that BAK1 endocytosis was accelerated when co-expressed with MSBP1. At least 20 cells were observed and fluorescence in each cell was calculated. The results were presented as average $\pm S D$. (C) Western blot analysis of extracts from $p 35 S: B A K 1$ p35S:MSBP1 and p35S:BAK1 transgenic plants using anti-HIS antibody showed the reduced amounts of BAK1 at membrane under MSBP1 overexpression. Soluble membrane proteins were extracted and equal amounts of proteins ( 35 $\mu \mathrm{g})$ were loaded. 

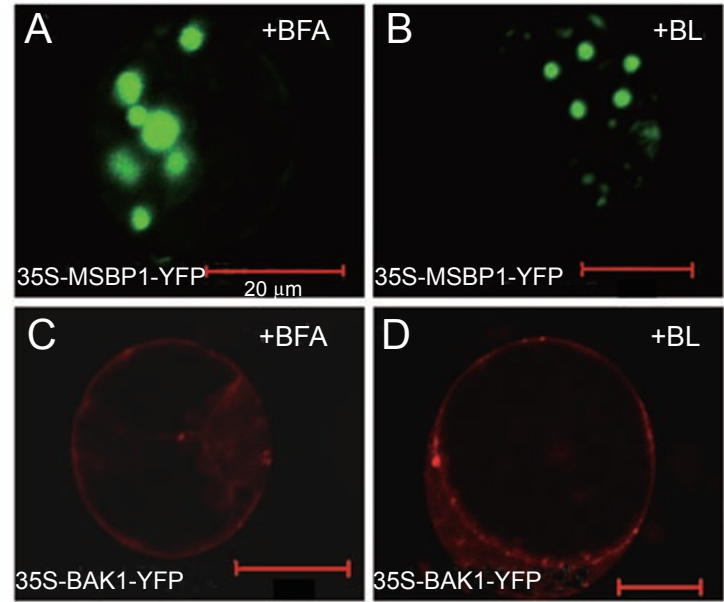

Figure 5 The subcellular localization of MSBP1 and BAK1 was not affected by BFA and BL. The localization of MSBP1 (A, B) and BAK1 (C, D) was observed after treatment with BFA (50 $\mu \mathrm{M})$ or $\mathrm{BL}(1 \mu \mathrm{M})$. Bar $=20 \mu \mathrm{m}$.

Consistent with the above observation, western blot analysis showed that the amount of membrane-localized BAK1 was decreased in $p 35 S: B A K 1$ p35S:MSBP1 plants when compared to that of $p 35 S: B A K 1$ (Figure 4C). However, discriminative localization of BAK1 was not observed in transgenic plants with suppressed expression of MSBP1 (through anti-sense approach, $p 35 S: A-M S B P 1$, Supplementary information, Figure S8). This might be due to the reason that the decreased expression of MSBP1 was not sufficient to change the localization of BAK1. Alternatively, it is likely due to the functional redundancy of MSBP1, which belongs to a small family with at least two closely related homologs in Arabidopsis [42].

Although it was shown that the vesicle trafficking was evidently increased in MSBP1-overexpressing plants and was suppressed in MSBP1-deficient plants, and p35S:MSBP1 plants were less sensitive to low concentrations of BFA in dark and less BFA compartments were formed, compared to that in WT [49], the localization of MSBP1 and BAK1 in Arabidopsis mesophyll protoplasts was not significantly affected by exogenous BFA or BL (Figure 5). This suggests that the internalization of MSBP1 is BFA- and BL-insensitive. Similar phenomenon was observed for the internalization of BRI1 and BAK1 in cowpea protoplasts [15]. As BFA mainly affects exocytosis, this result further confirms that MSBP1 regulates the endocytosis of BAK1.

MSBP1-BAK1 association does not affect the intensity of BRI1-BAK1 interaction, but inhibits the BRI1-BAK1 association in vivo

To test whether MSBP1-BAK1 interaction affects the BRI1-BAK1 association and hence suppresses BR signaling, we performed a yeast-based three-hybrid experiment. As shown in Figure 6, induced expression of MSBP1 protein (Figure 6A) did not reduce the BRI1-

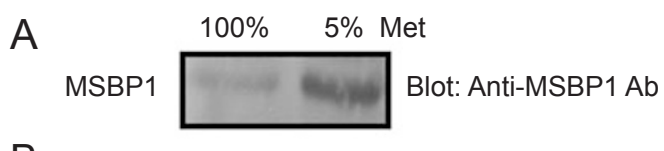

B

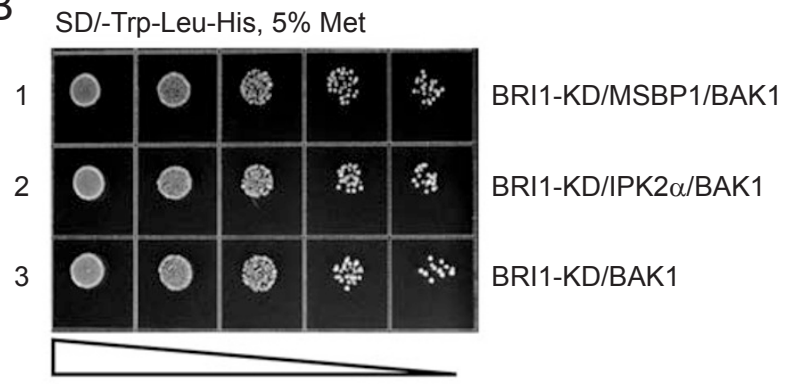

C

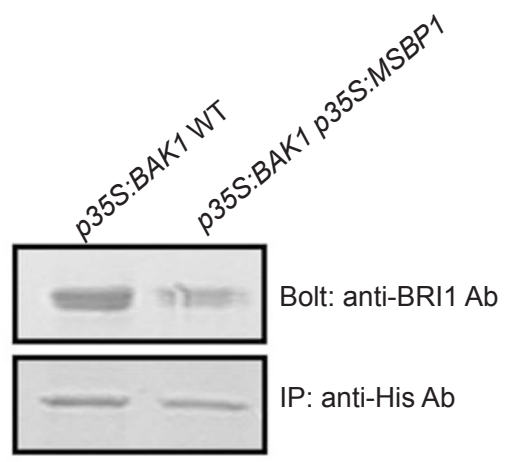

Figure 6 MSBP1-BAK1 association does not influence the interaction intensity of BRI1 and BAK1, but inhibits the BRI1-BAK1 association in vivo. (A) The inducible expression of MSBP1 under low amounts of Met. Western blot analysis confirmed the expression of MSBP1 in yeast cells under low concentrations of Met. Yeast cells grown on the SD-Leu/-Trp/-His dropout medium supplemented with $134 \mu \mathrm{M}$ (left) or $6.7 \mu \mathrm{M}$ (right) methionine were employed for inducible expressions of MSBP1 proteins. Soluble proteins were extracted and equal amount of proteins $(\sim 15 \mu \mathrm{g})$ were used for western blot analysis. The protein concentration was quantified by the Bradford method [62]. (B) Yeast-based three-hybrid analysis reveals that growth of yeast cells expressing MSBP1, BRI1-KD and BAK1 have no difference with those expressing IPK2 $\alpha$ (a negative control of MSBP1), BRI1-KD and BAK1, or BRI1-KD and BAK1. Coding regions of BRI1-KD are cloned in pBridge vector, and those of MSBP1 and IPK2 $\alpha$ are cloned in pBridge vector under the inducible promoter. The constructs are co-transformed into AH109 strains and growth of dosed yeast cells is observed. (C) Solubilized membrane proteins from p35S:BAK1 p35S:MSBP1 and $p 35 S: B A K 1$ plants were subjected to immunoprecipitation using anti-HIS antibody and immunoblot analysis using anti-BRI1 antibody. The BRI1-BAK1 interaction was decreased under MSBP1 overexpression. 
BAK1 association (Figure 6B), indicating that MSBP1BAK1 interaction does not affect the intensity of BRI1BAK1 interaction.

It was previously shown that increased endosomal localization of BRI1 could enhance BR signaling and genomic responses [35], and BAK1 accelerates BRI1 endocytosis. As MSBP1-BAK1 interaction enhances BAK1 endocytosis, it is reasonable to hypothesize that BAK1BRI1 association at membrane will be reduced under MSBP1 overexpression. Indeed, immuno-precipitation analysis showed that less BRI1 proteins were associated with BAK1 in p35S:MSBP1 plants than that in WT (Figure $6 \mathrm{C}$ ), revealing the suppressed BAK1-BRI1 association under MSBP1 overexpression. As heterodimerization and sequential transphosphorylation of BRI1/BAK1 enhance the signaling output $[13,50]$, the MSBP1-BAK1 interaction would inhibit BRI1-BAK1 association at the plasma membrane, resulting in the decreased heterodimerization of BRI1-BAK1 and subsequently reduced endosomal localization of BRI1, and finally suppressed BR signaling.

\section{Discussion}

MSBP1-BAK1 interaction may play an important role in ligand sensing

The LRR domain in the extracellular region of both BRI1 and BAK1 is thought to mediate protein-protein interactions [51, 52]. However, no protein interacting with these regions was identified up to now, although the BRI1 extracellular domain is able to bind BL molecules [10]. The fact that MSBP1 could bind BL and modulate plant responses to $\mathrm{BL}$ suggests the possible roles of MSBP1 in BR signaling. Here, we demonstrated that MSBP1 could interact with the extracellular LRR domain of BAK1 to regulate BR signaling.

The bril mutant that had a mutation in the extracellular region showed BR-insensitive phenotypes and the chimeric receptor harboring the BRI1-LRR and the kinase domain of XA21 could initiate plant defense responses when treated with $\mathrm{BL}$, indicating that the extracellular region of BRI1 mediates the positive regulatory effects on its intracellular kinase domain through binding the ligand molecules [8]. rpk1 mutant showed ABA insensitivity and plants overexpressing RPK1-LRR (including the transmembrane domain) were insensitive to ABA in seedling growth and seed germination, revealing that overexpressed LRR domain has a dominantnegative effect on RPK1 [53]. These studies suggest that extracellular regions of RLKs (serine/threonine receptorlike kinases) play a prominent role in their functions. In addition, the 70-amino acid island domain and carboxy- terminal flanking LRR in the extracellular domain of BRI1 could directly bind active BRs [10]; however, there has been no report showing that BAK1 binds to ligand directly. Our results suggest that MSBP1-BAK1 interaction might be another BR sensing mechanism independent of BRI1, supported by the facts that MSBP1 could bind $\mathrm{BL}$ and modulate plant responses to $\mathrm{BL}$.

In yeast, the BAK1/BRI1 transphosphorylation activity is interdependent, since neither of them can be phosphorylated if the other one lacks the kinase activity. However, the recombinant polypeptides of their cytosolic parts can interact with and transphosphorylate each other, even when one partner is inactive, albeit with reduced efficiency $[11,46]$. These results indicate that the intracellular domains act more independently than the full-length proteins. Accordingly, our results showed that the intracellular kinase domain of BAK1 has greater stimulating effects on BR signaling (Figure 2A and 2B).

$M S B P 1$ represses $B R$ signaling by stimulating $B A K 1$ endocytosis

Preliminary studies by yeast-based three-hybrid analysis showed that MSBP1-BAK1 association did not influence the intensity of BAK1-BRI1 interaction; however, MSBP1-BAK1 association did affect the quantity of BAK1-BRI1 interaction in vivo. As BRI1 could form homodimers at plasma membrane and BAK1-BRI1 heterodimerization was not uniformly distributed at plasma membrane, the increased BAK1 endocytosis caused by its association with MSBP1 will result in a reduction of BRI1 endocytosis stimulated by BAK1 and thus repressed BR signaling (Figure 7). Based on the different binding affinities to BR molecules, of which BRI1 is

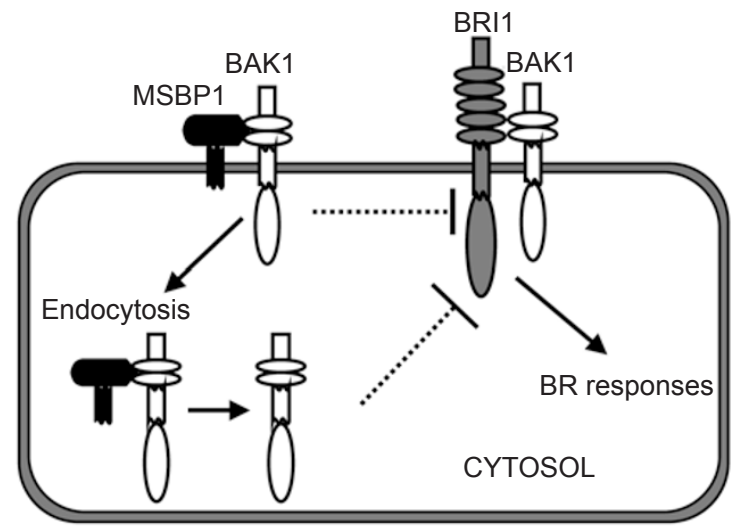

Figure 7 Hypothetical model for negative roles of MSBP1 in BR signaling. MSBP1-BAK1 interaction at plasma membrane significantly stimulates BAK1 endocytosis and reduces the BRI1BAK1 association, resulting in suppressed BR signaling. 
much higher than MSBP1, it is likely that MSBP1 will mainly function as a negative regulator under high concentrations of BR in the early step of BR signal transduction.

The MSBP1-BAK1 association was not influenced by ligand binding, which is consistent with the observation that ligand does not affect the accelerated endocytosis of BAK1 caused by the interaction with MSBP1, suggesting that ligand binding does not contribute much to BAK1 endocytosis. However, other possibilities for the negative regulatory role of MSBP1 in BR signaling cannot be excluded. MSBP1 may function as a BR carrier to regulate the $\mathrm{BR}$ amounts, or control BR homeostasis independently of BAK1 endocytosis.

MSBP1 involves in BR-mediated photomorphogenesis through both BAK1-dependent and-independent pathways

BR promotes hypocotyl elongation in light; while in dark, lower concentrations of BR promote and higher ones inhibit the hypocotyl elongation $[4,54,55]$. As a negative regulator of $\mathrm{BR}$ signaling, $M S B P 1$ expression in the hypocotyl is stimulated by light, and inhibited by dark [42]. Although the interaction between ELG and MSBP1 was reduced compared to the normal BAK1, overexpression of MSBP1 in elg mutant only suppressed its hypersensitive response to BR under darkness, but did not inhibit hypocotyl elongation or alter sensitivity of elg seedlings to BR in light (Figure 3B). Additionally, overexpression of BAK1 did not obviously inhibit the expression of $C P D$ gene in the $M S B P 1$-overexpressing seedlings (Figure 2C). These results indicate that the elongated hypocotyls of elg in light are not due to the reduced MSBP1-BAK1 interaction and MSBP1 might be involved in BR-mediated photomorphogenesis through both BAK1-dependent and -independent pathways.

In summary, MSBP1 could specifically interact with the extracellular domain of BAK1 in vivo in a BL-independent manner to stimulate the endocytosis of BAK1, resulting in suppressed BR signaling. Recent studies have raised the possibility to increase crop yield and improve crop quality by modulating BR signaling or biosynthesis [56]. MSBP1, because of its role as a negative regulator in BR signaling, can be one such candidate that might be useful in modifying BR sensitivity for crop improvement.

\section{Materials and Methods}

\section{Plant materials and growth conditions}

Arabidopsis thaliana ecotype Columbia (Col) was used as WT control. Col and MSBP1-overexpressing plants (p35S:MSBP1) were used to generate plants overexpressing intracellular kinase domain of BAK1 (p35S:BAK1-KD, p35S:BAK1-KD p35S:MSBP1) or whole BAK1 (p35S:BAK1, p35S:BAK1 p35S:MSBP1). Antisense MSBP1 transgenic plants ( $p 35 S: A-M S B P 1)$ were used for physiological analysis. bak1 mutant (SALK_116202, Col-0 background; or CS6125, Ws background) was used. Ecotype Landsberg erecta (Ler) was used for comparison with elg mutant. For measurement of root and hypocotyl lengths, seeds were sterilized and germinated on MS medium supplemented with sucrose $(2 \%, \mathrm{~W} /$ $\mathrm{V})$ and bacto-agar $(1 \%, \mathrm{~W} / \mathrm{V})$, with or without $\mathrm{BL}$, and vertically grown under dark for 4 days or light (16h light/ 8h dark) for 7 days. The seedlings were photographed and lengths of root and hypocotyl were measured by ImageJ software.

\section{Two-hybrid and three-hybrid analysis in yeast cells}

Whole coding region of MSBP1 was amplified using primers p1 (5'-CCG GAATTC ATG GCG TTA GAA CTA TGG CA3', added EcoRI site underlined) and p2 (5'-GCGGATCCT ACT CCT CCT TCT TCA ACA-3', added BamHI site underlined), and subcloned into vectors pGBKT7 or pGADT7. Full-length BAK1 was PCR amplified with primers p3 (5'-TCT GAATTC ATG GAA CGA AGA TTA ATG ATC-3', added EcoRI site underlined) and p4 (5'-CGC GGATCC TTA TCT TGG ACC CGA GGG GTA3', added BamHI site underlined), and subcloned into vector pGADT7. Primers p3 and p5 (5'-CGC GAGCTC CTG GTA CAT AAA AGA AGT GG-3', SacI site underlined) were used to amplify the extracellular region of BAK1. Primers p6 (5'-TCT $\underline{\text { GAATTC }}$ ATG GAC CCA GAA GTT CAT TTA GG-3', added EcoRI site underlined) and p7 (5'-CGC GGATCC TTA CCA CTC TTC CCA TCT CTC AGC TA-3', added BamHI site underlined) were used to amplify the intracellular region of BAK1. Primers p8 (5'-CAT GCCATGGAG CCA TCT CAG TCT TTA TAC AGA G-3', added NcoI site underlined) and p9 (5'-CGGGATCCC AAT CCC ATC GCC ACA CTA CCA-3', added BamHI site underlined) were used to amplify the extracellular region of BRI1; p10 (5'-GGAATTCAG AGA GAT GAG GAA GAG AC-3', added EcoRI site underlined) and p11 (5'-CGGGATCCT CAT AAT TTT CCT TCA GGA AC-3', added $B a m H I$ site underlined) were used to amplify the intracellular region of BRI1. The resultant PCR products were individually subcloned into vectors pGADT7 or pGBKT7 that precut with appropriate enzymes. The extracellular domain of Xa21 was subcloned into vector pGADT7 with primers Xa21-1 (5'-CCG GAATTC ATG GCG CGG GCA ATG ATG TTG-3', added EcoRI site underlined) and Xa21-2 (5'-GCGGATCCA ACT AGC TCT CCT TTG TAC AC-3', added Bam HI site underlined). Obtained constructs were sequenced to ensure no nucleotide change or substitution during PCR amplification. Desired pairs of constructs were co-transformed into AH109 yeast cells to examine the interactions of relevant proteins. The positive clones were identified by the criteria that yeast cells can grow on SD-Trp/-Leu/-His/-Ade medium and have $\beta$-galactosidase activities.

LexA yeast two-hybrid system was employed to calculate the relative $\beta$-galactosidase activities. Coding regions of MSBP1 (amplified by $\mathrm{p} 1$ and $\mathrm{p} 2$ ) and BRI1-KD (amplified by p10 and p11) were subcloned into vector pEG202; and those of BAK1 and mBAK1 (amplified by p3 and p12, 5'-ACT TCTCGAGTT ATC TTG GAC CCG AGG GGT A-3', added XhoI site underlined) were subcloned into vector pJG4-5, and used as positive and negative controls, respectively. Yeast transformation and calculation of relative $\beta$-galactosidase activities were performed as described 
[57]. At least 10 clones were randomly selected for activity measurement. The yeast cells were inoculated on induction synthetic defined plates containing 5-bromo-4-chloro-3-indoyl-b-D-galactopyranoside $(20 \mathrm{mg} / \mathrm{ml})$, grown at $30^{\circ} \mathrm{C}$ for $13 \mathrm{~h}$ with supplemented $\mathrm{BL}$ at different concentrations.

cDNA region coding for intracellular kinase domain of BRI1 (BRI1-KD, amplified by $\mathrm{p} 10$ and p11) was subcloned into the MCSI of pBridge ${ }^{\mathrm{TM}}$ vector (Clontech, Palo Alto, CA, USA). Fulllength MSBP1 (amplified by primers 5'-ATA AGA ATGCGGCCGCAA TGG CGT TAG AAC TAT GGC A-3', NotI site underlined; and 5'-GGA AGATCT CTA CTC CTC CTT CTT CAA CAC-3', $B g l$ II site underlined) and IPK2 $\alpha$ (amplified by primers $5^{\prime}$-ATA AGA ATGCGGCCGCAA TGC AGC TCA AAG TCC CTG-3', NotI site underlined; and 5'-GGA AGATCT CTA AGA ATC TGC AGA CTC ATC-3', BglII site underlined) were subcloned into the MCSII of pBridge ${ }^{\mathrm{TM}}$ vector. The obtained constructs were cotransformed into yeast cells harboring pGADT7-BAK1. MSBP1 and IPK $2 \alpha$ were conditionally expressed in response to the deficiency of methionone in the medium.

\section{Constructs and plant transformation}

HIS tag (6X histidine) was fused to C-terminus of BAK1 with primers p13 (5'-TCT TCTAGA ATG GCA GGG AGT AAT AGA ATT ACT GGA GCG-3', XbaI site underlined) and p14 (5'-TCT GAGCTC TTA GTG GTG GTG GTG GTG GTG TCT TGG ACC CGA GGG GTA TTC-3', SacI site underlined). Whole BAK1 was amplified with primers p15 (5'-TCT TCTAGA ATG GAA CGA AGA TTA ATG ATC-3', XbaI site underlined) and p14, and the amplified DNA fragments were subcloned into vector pATC940. The positive constructs were transformed into Arabidopsis by floral dipping method [58]. Resistant plants were selected through screening with appropriate antibiotics (hygromycin or kanamycin), and the confirmed transformants were transplanted into soil for self-crossing. Homozygous T2 lines were used for further analysis.

Construct pCAMBIA1302-MSBP1-GFP was introduced into Arabidopsis previously [42]. The whole MSBP1 cDNA was amplified by primers (5'-CGC GTCGAC ATG GCG TTA GAA CTA TGG CA-3', SalI site underlined; and 5'-CGC GGATCC CTC CTC CTT CTT CAA CAC AG-3', BamHI site underlined) and sub-cloned into pGDR and pA7-YFP vectors. The whole BAK1 cDNA was amplified by primers (5'-CGC GTCGAC ATG GAA CGA AGA TTA ATG ATC-3', SalI site underlined; and p4) and sub-cloned into pGDR and pA7-CFP vectors; or by primers (5'CGT TCCATGGCG ATG GAA CGA AGA TTA ATG ATC3', NcoI site underlined; and 5'-CGC AGATCT ACT CTT GGA CCC GAG GGG TA-3', BglII site underlined) and sub-cloned into pCAMBIA1302 vector. All the resultant constructs were sequenced to confirm the accuracy of amplified fragments and then used for the transient expressions in Arabidopsis mesophyll protoplasts or onion cells, as described previously $[59,60]$.

\section{Antibodies}

Expression and purification of MSBP1 protein were performed as described previously [42]. The purified proteins were dialyzed and used for antibody generation (Antibody Research Center, SIBS). Rabbit was immunized, and specialization of antisera was analyzed by protein gel blot using protein extracts prepared from p35S:MSBP1 and WT plants.

His-Antibody, c-Myc-Antibody and HA-Antibody were from Santa Cruz Biotechnology (Santa Cruz, CA, USA).

\section{Co-IP and western blot analysis}

Protein extraction and Co-IP analysis of transformed yeast cells was performed as described [46]. HA tag and c-MYC tag are fused with GAL4 DNA activation domain (pGADT7) and GAL4 DNAbinding domain (pGBKT7), respectively. The yeast protein was supplemented with protease inhibitor cocktail (Sigma, St Louis, MO, USA), and mixed with protein A Sepharose CL-4B (Amersham Bioscience, Uppsala, Sweden) and appropriate antibody (HA or c-MYC monoclonal antibody, Santa Cruz Biotechnology) for immunoprecipitation $\left(4{ }^{\circ} \mathrm{C}\right.$, overnight $)$. The sepharose was washed and the resultant proteins were separated on a $10 \%$ SDSPAGE, which was then transferred to a polyvinylidene difluoride membrane (PerkinElmer, Boston, MA, USA) for western blot analysis. The blots were probed with the primary antibodies (diluted in TBST $(10 \mathrm{mM}$ Tris- $\mathrm{HCl}, 150 \mathrm{mM} \mathrm{NaCl}$, and $0.1 \%$ Tween 20 , $\mathrm{pH} 7.5)$ ), washed in TBST three times, then reacted with goat antirabbit or goat anti-mouse IgG conjugated with alkaline phosphatase (1:4 000 to 1:5 000; Santa Cruz Biotechnology) and visualized by developing with AP detection reagent (1:1 mixture of NBT and BCIP, Novagen, Madison, WI, USA).

Membrane fractions were isolated [9] and total soluble membrane proteins were extracted from 2-week-old Arabidopsis seedling grown on medium in the presence or absence of 24-epibrassinolide (100 nM, Sigma) for $8 \mathrm{~h}$. The immunoprecipitated proteins were analyzed by western blot using anti-MSBP1, anti-HIS-tag or anti-BRI1 antibodies.

\section{Quantitative real-time RT-PCR analysis}

Arabidopsis seedlings were grown on MS medium supplemented with $1 \mu \mathrm{M}$ BL or mock solution for 7 days, under $16 \mathrm{~h}$ light/8 h dark cycle. Total RNAs were extracted with TRIzol ${ }^{\mathrm{R}}$ reagent (Invitrogen, Carlsbad, CA, USA), incubated with DNAase (TaKaRa, Dalian, China) and reverse-transcribed (Toyobo, Osaka, Japan). Real-time RT-PCR analyses on the transcription of $C P D$ and ROT3 genes in Arabidopsis seedlings were carried out by using SYBR Green qPCR kit (Toyobo) with RotorGene 3000 system (Corbett Research, Sydney, Australia). The primers used were as follows: ACTIN2 (5'-TTC CCG TTC TGC GGT AGT GG-3' and 5'-CCG GTA TTG TGC TCG ATT CTG-3'); CPD (5'-TCA GAC GTG CAA TGA CGG ATG TTG-3' and 5'-AGA AGG GCC TGT CGT TAC CGA GTT-3'); ROT3 (5'-GAA TAT GGA GAT GAA GAG GCG T-3' and 5'-CAC GGA TCG AAT TGA TAG GGA-3'). The relative gene expression was presented as percentage $(\%) \pm$ standard deviation (SD) from independent biological repeats.

\section{Semi-quantitative RT-PCR analysis}

Total RNAs were extracted and semi-quantitative RT-PCR analyses were performed to study the expression levels of $B A K 1$ or $B A K 1-K D$ regions in transgenic Arabidopsis seedlings. The primers used were as follows: BAK1 (5'-CTT TGA CTG CTG TCC TGA CGC-3' and 5'-AAC TCC TCC CGC AAT CGC TCC A-3'); BAK1-KD (5'-CGA AAG ATT GCT TGT TTA TCC CTA C-3' and 5'-ACC ACG GCT TCA AAC TCT TCA T-3'); MSBP1 (5'-GCC CCT TCT TAT GGC TAT CAA A-3' and 5'-AGA CAG ATG CGG TTT CAG GTT C-3').

\section{Particle bombardment-mediated transient expression in onion epidermal cells \\ Transient expression in onion epidermal cells was performed with a Model PDS-1000/He Biolistic Particle Delivery System}


(BioRad, Hercules, CA, USA). The plasmids coated on gold particles were bombarded with the following parameters: 1100 psi rupture disc; $27 \mathrm{in}$. $\mathrm{Hg}$ vacuum and $6 \mathrm{~cm}$ distance from the stopping screen to the target tissues. After bombardment, onion pieces were incubated on solid MS medium at $25{ }^{\circ} \mathrm{C}$ under darkness for at least $24 \mathrm{~h}$.

For FM4-64 staining, onion epidermis was incubated in distilled water containing FM4-64 (final concentration $5 \mu \mathrm{M}$, Invitrogen) at room temperature. Stained samples were washed twice with water and then observed.

\section{SEM analysis}

Samples of WT and transgenic plants were collected from the 5 th foliage leaf of 3-week-old plants, and fixed in FAA solution overnight, then dehydrated with an ascending series of ethanol (50\%-100\%). Critical point drying was conducted in liquid carbon dioxide. Samples were further coated with gold-palladium and visualized under a JSM-6360LV scanning electron microscope (JEOL Ltd, Japan).

Transient expression in Arabidopsis mesophyll protoplasts Arabidopsis mesophyll protoplasts were prepared and transformed as described by Sheen [59]. The transformed protoplasts were stained by FM4-64 (final concentration $5 \mu \mathrm{M}$ ) [61] and incubated in protoplast medium for $0.5-1 \mathrm{~h}$. For BFA treatment, BFA (final concentration $50 \mu \mathrm{M}$, Sigma) was added to the culture medium and protoplasts were observed in intervals of $0.5-1 \mathrm{~h}$ after application. For BL treatment, $1 \mu \mathrm{M}$ (final concentration) was added to the culture medium.

\section{Fluorescence detection}

The fluorescence in transformed cells was detected with a Confocal Laser Scanning Microscope (Zeiss LSM 510 META, excitation wavelength $488 \mathrm{~nm}$ and $543 \mathrm{~nm}$ by argon laser, emission wavelength $505-530 \mathrm{~nm}$ and over $560 \mathrm{~nm}$ by BP filter; or excitation wavelength $458 \mathrm{~nm}$ and $514 \mathrm{~nm}$ by argon laser, emission wavelength $475-525 \mathrm{~nm}$ and $530-600 \mathrm{~nm}$ by BP filter). A total of 20-30 cells were imaged for each experiment. Coexpressed proteins in the same onion cells were detected in the same Pinhole. Mean intensity of fluorescence measurement was performed according to manufacturer's instruction and the ratio of cytoplasm and intact fluorescence intensity were calculated.

\section{Acknowledgments}

This study was supported by the Chinese Academy of Sciences and National Natural Science Foundation of China (Grants 30425029, 30421001, 90717001). We greatly thank Prof Hong Ma (Penn. State University, USA) for critical reading and writing improvement and Prof Nam-Hai Chua (The Rockefeller University, USA) for helpful comments. We thank the Salk Institute Genomic Analysis Laboratory for providing the sequence-indexed Arabidopsis T-DNA insertion mutants, and Prof Sheng Luan (University of California, Berkeley, USA) for providing the construct pATC940. We thank Prof Hong-Quan Yang (SIPPE, CAS) for providing LexA yeast two-hybrid system and Prof Zhi-Yong Wang (The Stanford University, USA) for providing the BRI1 antibody. We thank Mr Xiao-Shu Gao for the help on Confocal Laser Scanning Microscopy.

\section{References}

1 Clouse SD, Sasse JM. BRASSINOSTEROIDS: essential regulators of plant growth and development. Annu Rev Plant Physiol Plant Mol Biol 1998; 49:427-451.

2 Takahashi T, Gasch A, Nishizawa N, Chua N. The DIMINUTO gene of Arabidopsis is involved in regulating cell elongation. Genes Dev 1995; 9:97-107.

3 Clouse SD, Langford M, Mcmorris TC. A brassinosteroidinsensitive mutant in Arabidopsis thaliana exhibits multiple defects in growth and development. Plant Physiol 1996; 111:671-678.

4 Li J, Nagpal P, Vitart V, McMorris TC, Chory J. A role for brassinosteroids in light-dependent development of Arabidopsis. Science 1996; 272:398-401.

5 Szekeres M, Nemeth K, Koncz-kalman Z, et al. Brassinosteroids rescue the deficiency of CYP90, a cytochrome P450, controlling cell elongation and de-etiolation in Arabidopsis. Cell 1996; 85:171-182.

6 Li J, Nam KH, Vafeados D, Chory J. BIN2, a new brassinosteroids insensitive locus in Arabidopsis. Plant Physiol 2001; 127:14-22.

7 Li J, Chory J. A putative leucine-rich repeat receptor kinase involved in brassinosteroid signal transduction. Cell 1997; 90:929-938.

8 He ZH, Wang ZY, Li J, et al. Perception of brassinosteroids by the extracellular domain of the receptor kinase BRI1. Science 2000; 288:2360-2363.

9 Wang ZY, Seto H, Fujioka S, Yoshida S, Chory J. BRI1 is a critical component of a plasma-membrane receptor for plant steroids. Nature 2001; 410:380-383.

10 Kinoshita T, Cano-Delgado A, Seto H, et al. Binding of brassinosteroids to the extracellular domain of plant receptor kinase BRI1. Nature 2005; 433:167-171.

11 Li J, Wen J, Lease KA, Doke JT, Tax FE, Walker JC. BAK1, an Arabidopsis LRR receptor-like protein kinase, interacts with BRI1 and modulates brassinosteroid signaling. Cell 2002; 110:213-222.

12 Li J, Nam KH. Regulation of brassinosteroids signaling by a GSK3/SHAGGY-like kinase. Science 2002; 295:1299-1301.

13 Wang X, Li X, Meisenhelder J, et al. Autoregulation and homodimerization are involved in the activation of the plant steroid receptor BRI1. Dev Cell 2005; 8:855-865.

14 Wang X, Goshe MB, Soderblom EL, et al. Identification and functional analysis of in vivo phosphorylation sites of the Arabidopsis BRASSINOSTEROID-INSENSITIVE 1 receptor kinase. Plant Cell 2005; 17:1685-1703.

15 Russinova E, Borst JW, Kwaaitaal M, et al. Heterodimerization and endocytosis of Arabidopsis brassinosteroids receptors BRI1 and AtSERK3 (BAK1). Plant Cell 2004; 16:32163229.

16 Tang W, Kim TW, Oses-Prieto JA, et al. BSKs mediate signal transduction from the receptor kinase BRI1 in Arabidopsis. Science 2008; 321:557-560.

17 Wang X, Chory J. Brassinosteroids regulate dissociation of BKI1, a negative regulator of BRI1 signaling, from the plasma membrane. Science 2006; 313:1118-1122.

18 Wang ZY, Nakano T, Gendron J, et al. Nuclear-localized 
BZR1 mediates brassinosteroids-induced growth and feedback suppression of brassinosteroids biosynthesis. Dev Cell 2002; 2:505-513.

19 Zhao J, Peng P, Schmitz RJ, Decker AD, Tax FE, Li J. Two putative BIN2 substrates are nuclear component of brassinosteroid signaling. Plant Physiol 2002; 13:1221-1229.

20 Yin Y, Wang ZY, Mora-Garcia S, et al. BES1 accumulates in the nucleus in response to brassinosteroids to regulate gene expression and promote stem elongation. Cell 2002; 109:181191.

21 Yin Y, Vafeados D, Tao Y, Yoshida S, Asami T, Chory J. A new class of transcription factors mediates brassinosteroidregulated gene expression in Arabidopsis. Cell 2005; 120:249259.

22 Vert G, Chory J. Downstream nuclear event in brassinosteroids signaling. Nature 2006; 441:96-100.

23 He JX, Gendron JM, Yang Y, Li J, Wang ZY. The GSK3-like kinase BIN2 phosphorylates and destabilizes BZR1, a positive regulator of the brassinosteroid signaling pathway in Arabidopsis. Proc Natl Acad Sci USA 2002; 99:10185-10190.

24 He JX, Gendron JM, Sun Y, et al. BZR1 is a transcriptional repressor with dual roles in brassinosteroid homeostasis and growth response. Science 2005; 307:1634-1638.

25 Edwards PA, Ericsson J. Sterols and isoprenoids: signaling molecules derived from the cholesterol biosynthetic pathway. Annu Rev Biochem 1999; 68:157-185.

26 Michaux G, Gansmuller A, Hindelang C, Labouesse M. CHE14 , a protein with a sterol-sensing domain, is required for apical sorting in C. elegans ectodermal epithelial cells. Curr Biol 2000; 10:1098-1107.

27 Ingham PW. Hedgehog signaling: a tale of two lipids. Science 2001; 294:1879-1881.

28 Geldner N, Friml J, Stierhof YD, Jurgens G, Palme K. Auxin transport inhibitors block PIN1 cycling and vesicle trafficking. Nature 2001; 413:425-428.

29 Friml J, Wisniewska J, Benkova E, Mendgen K, Palme K. Lateral relocation of auxin efflux regulator PIN3 mediates tropism in Arabidopsis. Nature 2002; 415:806-809.

30 Dhonukshe P, Aniento F, Hwang I, et al. Clathrin-mediated constitutive endocytosis of PIN auxin efflux carriers in Arabidopsis. Curr Biol 2007; 17:520-527.

31 Gómez-Gómez L, Boller T. FLS2: an LRR receptor-like kinase involved in the perception of the bacterial elicitor flagellin in Arabidopsis. Mol Cell 2000; 5:1003-1011.

32 Robatzek S, Chinchilla D, Boller T. Ligand-induced endocytosis of the pattern recognition receptor FLS2 in Arabidopsis. Genes Dev 2006; 20:537-542.

33 Chinchilla D, Zipfel C, Robatzek S, et al. A flagellin-induced complex of the receptor FLS2 and BAK1 initiates plant defence. Nature 2007; 448:497-500.

34 Heese A, Hann DR, Gimenez-Ibanez S, et al. The receptorlike kinase SERK3/BAK1 is a central regulator of innate immunity in plants. Proc Natl Acad Sci USA 2007; 104:1221712222 .

35 Geldner N, Hyman DL, Wang XL, Schumacher K, Chory J. Endosomal signaling of plant steroid receptor kinase BRI1. Genes Dev 2007; 21:1598-1602.

36 Kwaaitaal MA, de Vries SC, Russinova E. Arabidopsis thaliana somatic embryogenesis receptor kinase 1 protein is present in sporophytic and gametophytic cells and undergoes endocytosis. Protoplasma 2005; 226:55-65.

37 Karlova R, Boeren S, Russinova E, Aker J, Vervoort J, de Vries S. The Arabidopsis SOMATIC EMBRYOGENESIS RECEPTOR-LIKE KINASE1 protein complex includes BRASSINOSTEROID-INSENSITIVE1. Plant Cell 2006; 18:626-638.

38 Evans RM. The steroid and thyroid hormone receptor superfamily. Science 1988; 240:889-895.

39 Glass CK. Differential recognition of target genes by nuclear receptor monomers, dimers, and heterodimers. Endocr Rev 1994; 15:391-407.

40 Beato M, Herrlich P, Schutz G. Steroid hormone receptors: many actors in search of a plot. Cell 1995; 83:851-857.

41 Mangelsdorf DJ, Thummel C, Beato M, et al. The nuclear receptor superfamily: the second decade. Cell 1995; 83:835839.

42 Yang XH, Xu ZH, Xue HW. Arabidopsis membrane steroidbinding protein 1 is involved in inhibition of cell elongation. Plant Cell 2005; 17:116-131.

43 Sonnhammer ELL, von Heijne G, Krogh A. A hidden Markov model for predicting transmembrane helices in protein sequences. Proceedings of the Sixth International Conference on Intelligent Systems for Molecular Biology. Menlo Park, CA: AAAI Press, 1998:175-182.

44 Krogh A, Larsson B, von Heijne G, Sonnhammer ELL. Predicting transmembrane protein topology with a hidden Markov model: Application to complete genomes. J Mol Biol 2001; 305:567-580.

45 Song WY, Wang GL, Chen LL, et al. A receptor kinase-like protein encoded by the rice disease resistance gene, Xa21. Science 1995; 270:1804-1806.

46 Nam KH, Li J. BRI1/BAK1, a receptor kinase pair mediating brassinosteroid signaling. Cell 2002; 110:203-212.

47 Whippo CW, Hangarter RP. A brassinosteroids-hypersensitive mutant of BAK1 indicates that a convergence of photomorphogenic and hormonal signaling modulates phototropism. Plant Physiol 2005; 139:448-457.

$48 \mathrm{Xu} \mathrm{J}$, Scheres B. Dissection of Arabidopsis ADP-RIBOSYLATION FACTOR 1 function in epidermal cell polarity. Plant Cell 2005; 17:525-536.

49 Yang X, Song L, Xue HW. Membrane steroid binding protein 1 (MSBP1) stimulates tropism by enhancing polar auxin transport. Mol Plant 2008; 1:1077-1087.

50 Wang X, Kota U, He K, et al. Sequential transphosphorylation of the BRI1/BAK1 receptor kinase complex impacts early events in brassinosteroid signaling. Dev Cell 2008; 15:220235.

51 Kobe B, Deisenhofer J. A structural basis of the interactions between leucine-rich repeats and protein ligands. Nature 1995; 374:183-186.

52 Jones DA, Jones JDG. The role of leucine-rich repeat proteins in plant defences. Adv Bot Res 1997; 24:89-167.

53 Osakabe Y, Maruyama K, Seki M, Satou M, Shinozaki K, Yamaguchi-Shinozaki K. Leucine-rich repeat receptor-like kinase 1 is a key membrane-bound regulator of abscisic acid early signaling in Arabidopsis. Plant Cell 2005; 17:11051119.

54 Ephritikhine G, Pagant S, Fujioka S, et al. The sax1 mutation 
defines a new locus involved in the brassinosteroid biosynthesis pathway in Arabidopsis thaliana. Plant J 1999; 18:315320.

55 De Grauwe L, Vandenbussche F, Tietz O, Palme K, Van Der Straeten D. Auxin, ethylene and brassinosteroids: tripartite control of growth in the Arabidopsis hypocotyl. Plant Cell Physiol 2005; 46: 827-836.

56 Bishop GJ. Brassinosteroid mutants of crops. J Plant Growth Regul 2003; 22:325-335.

57 Yang HQ, Tang RH, Cashmore AR. The signaling mechanism of Arabidopsis CRY1 involves direct interaction with COP1. Plant Cell 2001; 13:2573-2587.

58 Clough SJ, Bent AF. Floral dip: a simplified method for Agrobacterium-mediated transformation of Arabidopsis thali- ana. Plant J 1998; 16: 735-743.

59 Sheen J. Signal transduction in maize and Arabidopsis mesophyll protoplasts. Plant Physiol 2001; 127: 1466-1475.

60 Goodin MM, Dietzgen RG, Schichnes D, Ruzin S, Jackson AO. pGD vectors: versatile tools for the expression of green and red fluorescent protein fusions in agroinfiltrated plant leaves. Plant $J$ 2002; 31:375-383.

61 Shah K, Russinova E, Gadella TW Jr, Willemse J, De Vries SC. The Arabidopsis kinase-associated protein phosphatase controls internalization of the somatic embryogenesis receptor kinase 1. Genes Dev 2002; 16:1707-1720.

62 Bradford MM. A rapid and sensitive for the quantitation of microgram quantities of protein utilizing the principle of protein-dye binding. Anal Biochem 1976; 72:248-254.

(Supplementary information is linked to the online version of the paper on the Cell Research website.) 